\title{
Nucleosome organization in the vicinity of transcription factor binding sites in the human genome
}

\author{
Yumin Nie, Xiangfei Cheng, Jiao Chen and Xiao Sun ${ }^{*}$
}

\begin{abstract}
Background: The binding of transcription factors (TFs) to specific DNA sequences is an initial and crucial step of transcription. In eukaryotes, this process is highly dependent on the local chromatin state, which can be modified by recruiting chromatin remodelers. However, previous studies have focused mainly on nucleosome occupancy around the TF binding sites (TFBSs) of a few specific TFs. Here, we investigated the nucleosome occupancy profiles around computationally inferred binding sites, based on 519 TF binding motifs, in human GM12878 and K562 cells.

Results: Although high nucleosome occupancy is intrinsically encoded at TFBSs in vitro, nucleosomes are generally depleted at TFBSs in vivo, and approximately a quarter of TFBSs showed well-positioned in vivo nucleosomes on both sides. RNA polymerase near the transcription start site (TSS) has a large effect on the nucleosome occupancy distribution around the binding sites located within one kilobase to the nearest TSS; fuzzier nucleosome positioning was thus observed around these sites. In addition, in contrast to yeast, repressors, rather than activators, were more likely to bind to nucleosomal DNA in the human cells, and nucleosomes around repressor sites were better positioned in vivo. Genes with repressor sites exhibiting well-positioned nucleosomes on both sides, and genes with activator sites occupied by nucleosomes had significantly lower expression, suggesting that actions of activators and repressors are associated with the nucleosome occupancy around their binding sites. It was also interesting to note that most of the binding sites, which were not in the DNase I-hypersensitive regions, were cell-type specific, and higher in vivo nucleosome occupancy were observed at these binding sites.

Conclusions: This study demonstrated that RNA polymerase and the functions of bound TFs affected the local nucleosome occupancy around TFBSs, and nucleosome occupancy patterns around TFBSs were associated with the expression levels of target genes.
\end{abstract}

Keyword: Nucleosome occupancy, Transcription factor binding site, Clustering

\section{Background}

Transcription factors (TFs) bind to specific DNA sequences and interact with components of the RNA polymerase complex, or with other complexes, to regulate transcription in a cell type-specific manner, and this process is highly dependent on the chromatin structure in eukaryotes [1-3]. The basic unit of chromatin structure is the nucleosome, consisting of histone octamers wrapped in 147 base pairs (bps) of DNA [4,5]. Eukaryotic genomic DNA is assembled into nucleosomes and is

\footnotetext{
* Correspondence: xsun@seu.edu.cn

State Key Laboratory of Bioelectronics, School of Biological Science \& Medical Engineering, Southeast University, 210096 Nanjing, China
}

further packaged into chromatin to achieve high compaction. Nucleosomes can directly regulate the accessibility of TFs and transcriptional machinery to the DNA sequences [6]. Sequences in nucleosome-depleted regions are easier to access, while the accessibility of DNA within nucleosomes depends on nucleosome dynamics $[7,8]$. Although histone-DNA complexes are very stable, histones are constantly evicted and reassembled onto DNA templates in a locus-specific manner. Previous studies have suggested that promoters and other regulatory sequences are typically nucleosome-depleted, whereas transcribed regions tend to be occupied by wellpositioned nucleosomes, which are maintained by nucleosome-remodeling activities $[9,10]$. The occupancy 
patterns and dynamic positioning of nucleosomes thus play crucial roles in regulating eukaryotic transcription.

Nucleosomes influence the accessibility of TFs to DNA. TFs can, in turn, directly or indirectly recruit remodeling complexes, or other coregulators, to modify the local chromatin state. The binding of several TFs, such as the insulator binding protein CTCF [11,12], the RE1-silencing transcription factor (REST/NRSF) [13] and the multifunctional TF YY1 [14], has been suggested to initiate nucleosome depletion at TF binding sites (TFBSs) and the phased nucleosome arrays in the flanking regions in human cells. Nearly 3,000 TFs have been predicted computationally in the DNA-binding domain (DBD) database [15], and detailed manual curation has confirmed at least 1,400 TFs in the human genome [16]. However, chromatin immunoprecipitation followed by sequencing (ChIP-seq), a technique for measuring genomewide TF binding profiles, is only applied to one TF in a single experiment, making it difficult to identify binding locations for large numbers of factors in the specific cell type. Previous studies have, therefore, focused mainly on nucleosome occupancy around binding sites of a few specific TFs [11-13,17]. Computational methods have the advantage of being able to determine the accurate profiles for many factors in a specific sample $[18,19]$. Like many computational methods, CENTIPEDE [19], based on a hierarchical Bayesian mixture model, requires position weight matrices of known TF binding motifs; therefore, its ability is dependent on the availability of TF binding motifs. However, CENTIPEDE incorporates cell-specific experimental data to infer binding sites in a particular cell type, making it more accurate for predicting TFBSs.

To better understand the relationship between nucleosome positioning and TF binding, we focused on the CENTIPEDE-inferred binding sites for 519 TF binding motifs, representing up to a third of the human TF repertoire, and examined the nucleosome occupancy around these binding sites in human GM12878 and K562 cells. We further classified the binding sites by the distances of sites relative to the nearest gene and the functions of the bound TFs, to test whether the nucleosome occupancy exhibited distinct patterns. We finally clustered the nucleosome occupancy profiles around TFBSs and investigated their relevance to gene expression.

\section{Results}

\section{Nucleosome occupancy around TF binding sites}

Both in vitro and in vivo nucleosome occupancy data were used to examine the average nucleosome occupancy around the binding sites for 519 TF binding motifs (Additional file 1) in GM12878 and K562 cells. Nucleosomes can be assembled by genomic DNA and recombinant histones in the absence of cellular influences; therefore, in vitro nucleosome occupancy is affected mainly by the intrinsic specificity between a histone and the DNA sequences, whereas in vivo occupancy is influenced by sequence preferences, TFs and chromatin remodelers $[5,13,20]$. Analyses of in vivo data showed nucleosome-depleted regions at TFBSs and an array of well-positioned nucleosomes in the flanking regions (Figure 1A), which was consistent with the barrier model suggested in previous studies $[4,13]$. We then constructed in vitro nucleosome occupancy profiles, to test whether the in vivo nucleosome distributions were governed by the intrinsic sequence preferences of nucleosomes. We observed high in vitro nucleosome occupancy at TFBSs (Figure 1B), suggesting that binding sequences of TFs tend to form nucleosomes. We further analyzed the nucleotide composition of TF binding sequences and found that these DNA sequences were GC-rich (Figure 1C). Our analyses, which are consistent with previous studies [13,17], demonstrated that human TFBSs have high GC content and intrinsic nucleosome occupancy, but low in vivo nucleosome occupancy.

Low nucleosome signals in vivo are necessary for most TFBSs, as TFs may compete with nucleosomes to slide or evict them for access to the specific DNA [8]. It is generally believed that nucleosomes are depleted before TFs bind to their sites. However, a recent study argues that nucleosome eviction occurs after TF binding and, in fact, requires TF binding, suggesting that nucleosome loss may not be a prerequisite for TF binding [21]. The barrier model could explain the well-positioned nucleosomes around TFBSs. Binding of TFs can form barriers and other nucleosomes are stacked against them to generate the phased nucleosome arrays by ATP-dependent chromatin remodelers $[4,10]$. Despite DNA sequences encoding nucleosome occupancy at certain regions, TF binding can drive nucleosomes to occupy intrinsically unfavorable DNA sequences or evict nucleosomes from intrinsically favorable sites.

\section{Nucleosome occupancy around proximal and distal binding sites}

TFBSs may be located proximal or distal to the transcription start site (TSS). Binding sites in the core or proximal promoter are typically located within one kilobase $(\mathrm{kb})$, while distal sites may be situated up to several hundred $\mathrm{kb}$ from the core promoter [22]. Here, we defined proximal and distal sites as those located within $1 \mathrm{~kb}$ and beyond $10 \mathrm{~kb}$ from the nearest TSS, respectively, and investigated the in vivo nucleosome occupancy around these sites. The nucleosome occupancy profiles around distal sites in both GM12878 and K562 cells (Figure 2A and B) were similar to those around all binding sites, as described in Figure 1A. However, we observed lower in vivo nucleosome occupancy and fuzzier 

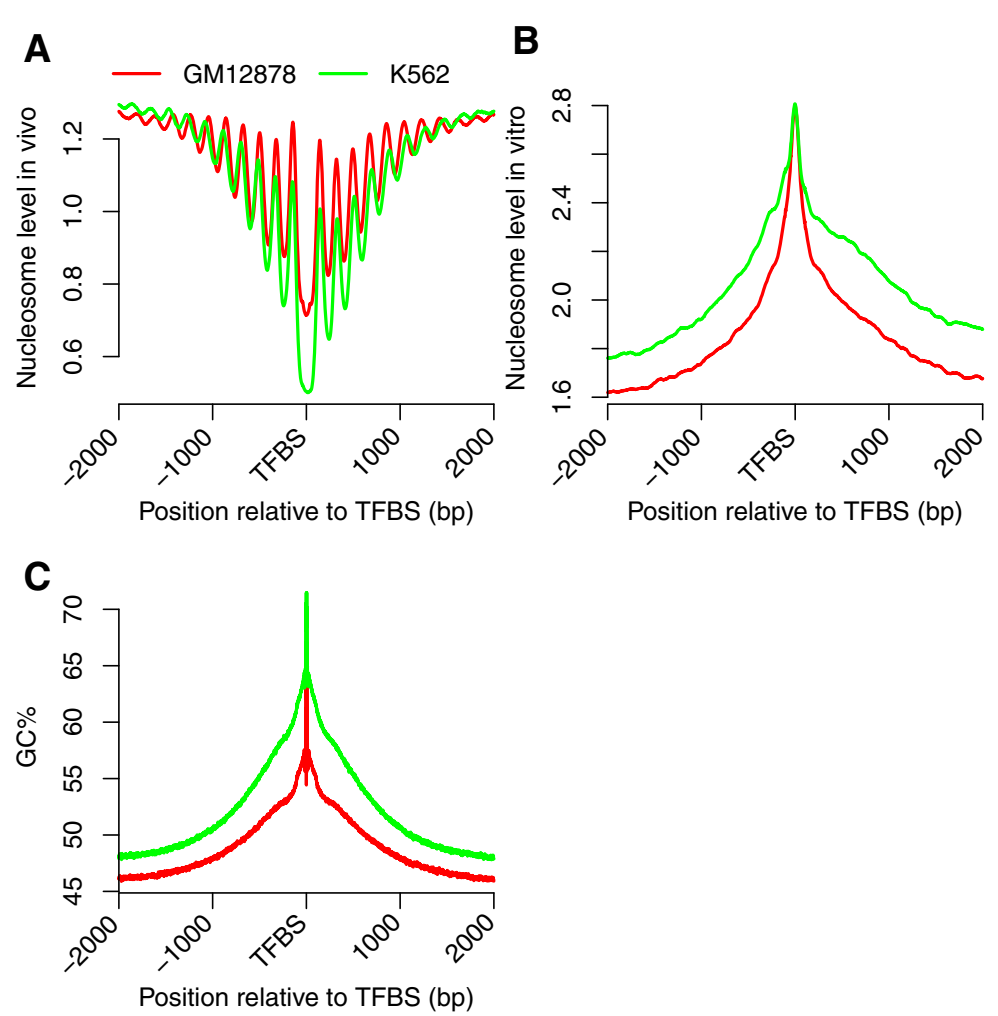

Figure 1 Nucleosome occupancy profiles and GC content around TF binding sites. (A) Nucleosomes were depleted at TFBSs and well positioned in the flanking regions in vivo. (B) High in vitro nucleosome occupancy was encoded at TFBSs. (C) High GC content was observed at TFBSs.

nucleosome positioning around proximal sites, which were more consistent with those around TSSs (Figure 2C). Nucleosome-depleted regions are also essential for TSSs, as RNA polymerase and a variety of auxiliary components bind to DNA and interact with each other around TSSs [4]. In order to explain the in vivo nucleosome occupancy pattern around the proximal sites, we divided proximal sites into 100-bp intervals based on their distances relative to the nearest TSS, and investigated the proportion of proximal sites in each interval. Our results indicated that $55.1 \%$ and $59.6 \%$ of proximal sites in GM12878 and K562 cells, respectively, were located within $200 \mathrm{bp}$ relative to TSSs (Figure 2D), suggesting that many proximal sites are located within nucleosome-depleted regions near TSSs, and nucleosome occupancy around proximal sites thus mostly reflect that around TSSs. Proximal sites fall within promoter regions near TSSs, where nucleosomes are generally depleted, and therefore the average nucleosome occupancy is lower at proximal sites and the nucleosome positioning around proximal sites is less pronounced. On the other hand, distal sites are far from promoters, where the nucleosome occupancy is higher, the binding of TFs in the distal regions is therefore more likely to recruit ATPdependent chromatin remodelers to generate the phased nucleosome arrays.

\section{Nucleosome occupancy around activator and repressor binding sites}

Activators and repressors positively and negatively regulate transcription, respectively. Both activators, such as Abf1 [23] and Reb1 [24] in yeast, and repressors, such as the REST protein [13] in human, have been suggested to bind to DNA sequences that intrinsically encode high nucleosome occupancy, and their binding will influence the nucleosome organization. In yeast, it has also been suggested that activator binding sites show significantly higher correlation with nucleosome sequence profiles compared with those of repressors [25]. Here, we queried the UniProt database to determine the functions of TFs [26], and investigated the nucleosome occupancy around activator and repressor binding sites to test whether the functions of bound TFs influenced the nucleosome occupancy distribution in human cells. Activators and repressors identified in GM12878 and K562 cells were listed in Additional file 2. We first examined the in vivo nucleosome occupancy around activator and repressor binding sites. Although nucleosome-depleted regions were observed at activator and repressor binding sites, nucleosomes around the repressors sites were better positioned compared with the activator sites in both GM12878 and K562 cells (Figure 3A). Repressor binding 


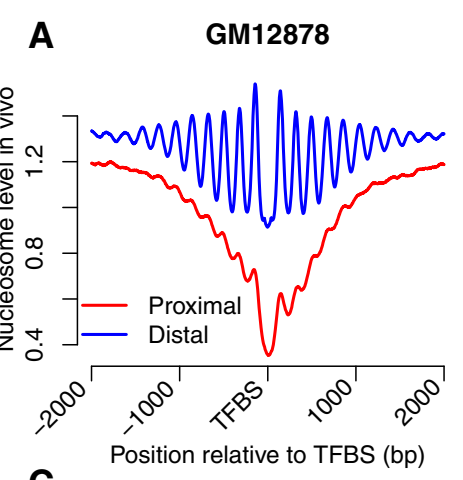

$c$

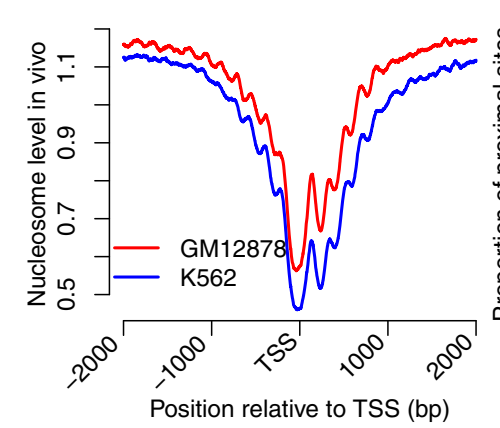

B $\quad \mathrm{K} 562$

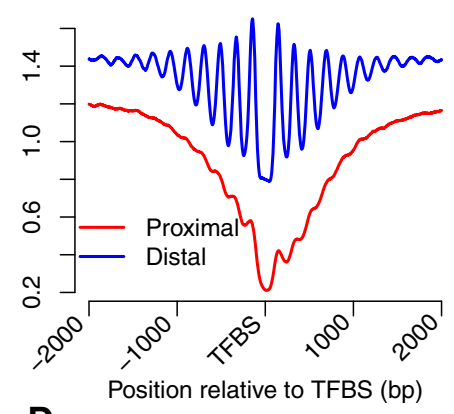

D

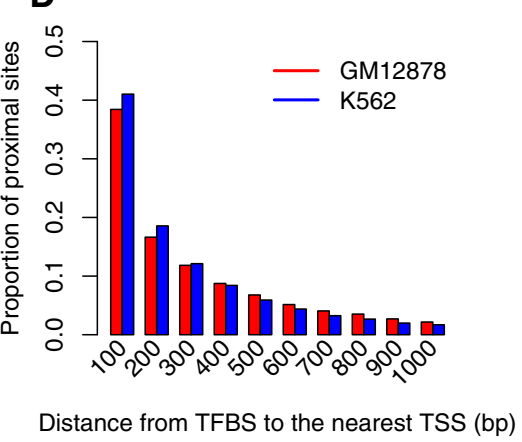

Figure 2 In vivo nucleosome occupancy profiles around proximal sites are similar with those around TSSs. (A, B) In vivo nucleosome occupancy profiles around the proximal and distal sites in both GM12878 and K562 cells. (C) In vivo nucleosome occupancy profiles around TSSs. (D) Proportion of proximal sites in each 100-bp interval.

sites are generally situated quite distally from TSSs, while multiple binding sites for activators are located in the promoter [22]. $55.0 \%$ of repressor sites and $44.9 \%$ of activators sites were located beyond $10 \mathrm{~kb}$ from TSS in the GM12878 cell line, while in the K562 cell line, the percentages of distal repressor and activator sites were $36.5 \%$ and $28.9 \%$, respectively. Therefore, we examined the in vivo nucleosome occupancy around the distal activator and repressor sites (Additional file 3) to avoid the influences of the transcriptional machinery near TSSs. Better-positioned nucleosomes were observed around the distal repressor sites (Figure 3B), indicating that repressor binding induced the nucleosome distribution. We further investigated the in vivo nucleosome occupancy around the distal sites for each of activators and repressors. Both activators, such as FLI1 and NFE2, and repressors, such as REST and Tel-2, can generate the phased nucleosome array (Additional file 4 and Additional file 5). However, the nucleosome positioning around REST binding sites was particularly obvious, which contributed largely to the better nucleosome positioning around repressor sites. It should be noted that for some repressors, such as Bcl6b_2, fuzzy nucleosome positioning was observed around their binding sites, suggesting that the formation of phased nucleosome array is associated with the specific TFs. We finally examined the in vitro nucleosome occupancy around activator and repressor binding sites.
Both activator and repressor sites were enriched in nucleosome sequence preferences (Figure 4A). However, unlike yeast, repressor binding sequences were more likely to intrinsically encode nucleosomes in the human genome, according to the analysis of the in vitro data (Figure 4B; Wilcoxon rank-sum test, $P<2.2 \times 10^{-16}$ ). The same conclusion was obtained from the analyses of distal sites. Repressor binding to sequences with higher in vitro nucleosome occupancy might have more elevated dependence on nucleosome dynamics and chromatin remodeling complexes [27,28], and this might contribute to the better-positioned nucleosomes flanking the repressor sites in vivo.

\section{Nucleosome occupancy around DNase I-hypersensitive and -resistant sites}

TFs compete with nucleosomes to access DNA and nucleosome depletion is thus generally observed at their binding sites. However, TFs can bind to DNA sequences with high in vivo nucleosome occupancy [5]. DNase I preferentially digests DNA in regions of low nucleosome occupancy and DNase I-hypersensitive regions reflect the accessibility of genome. We found that $10.8 \%$ and $3.1 \%$ of the binding sites in GM12878 and K562 cells, respectively, were not in DNase I-hypersensitive regions, and observed higher in vivo nucleosome occupancy at these DNase I-resistant binding sites, especially in the 

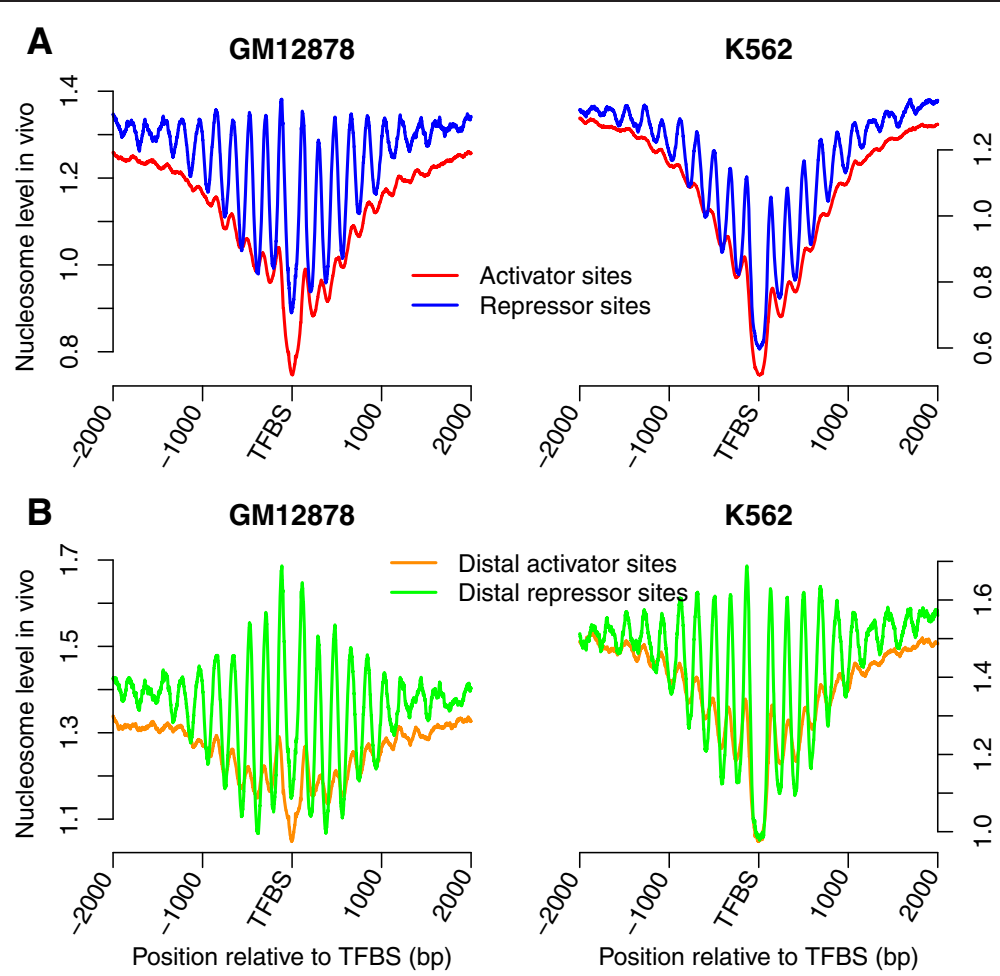

Figure 3 In vivo, nucleosomes are better positioned around repressor sites. (A) In vivo nucleosome occupancy profiles around the activator and repressor sites in both GM12878 and K562 cells. (B) In vivo nucleosome occupancy profiles around the distal activator and repressor sites.

K562 cell line (Figure 5). We then investigated the in vitro nucleosome occupancy around the DNase I-hypersensitive and -resistant sites. Similar in vitro nucleosome occupancy levels were observed around the DNase I-hypersensitive sites in GM12878 and K562 cells. However, for DNase Iresistant binding sites, in vitro nucleosome occupancy level in the K562 cell line was higher than that in the GM12878 cell line (Figure 6A). The same conclusions were obtained by analyzing the distal DNase I-hypersensitive and -resistant sites (Figure 6B). DNA sequences around DNase I-resistant sites in the K562 cell line were more likely to form nucleosomes, and TF binding in the DNase I-resistant regions had less effect on the local chromatin structure, which might contribute to the higher in vivo nucleosome occupancy around DNase Iresistant sites in the K562 cell line. CTCF, serum response factor SRF and c-Rel were three TFs that most frequently bound to DNase I-resistant regions in GM12878 cells, whereas the three TFs that most frequently bound to these regions were CTCF, SRF and HNF4 in K562 cells. CTCF is a multifunctional TF, while SRF and c-Rel are considered as activators. It was also interesting to note that most of the DNase Iresistant binding sites were cell-type specific. $90.5 \%$ and 81.5\% of these sites in GM12878 and K562 cells, respectively, were cell-type specific and were not bound by any TF in the other type of cells. These results suggested that the DNase I-resistant sites might play important roles in the specific cells.

\section{Clustering nucleosome occupancy around TF binding sites}

Some TFs can bind to the DNase I-resistant regions with high in vivo nucleosome occupancy and might regulate gene expression in a cell-type-specific way. A traditional profile that averages over all TF binding sites will neglect these specific nucleosome occupancy patterns. Therefore we applied an unsupervised clustering method called Clustered AGgregation Tool (CAGT) [29], to discover the diverse in vivo nucleosome occupancy patterns around TFBSs. The cluster analysis of all TFBSs indicated that only $22.6 \%$ and $25.2 \%$ of TFBSs in GM12878 and K562 cells, respectively, were flanked by wellpositioned nucleosomes on both sides (Figure 7). The cluster analysis of proximal and distal binding sites further indicated that TFBSs exhibiting symmetric positioning of nucleosomes were mainly located in the distal regions (Additional file 6). We clustered the nucleosome signals around distal activator and repressor binding sites and found that higher proportions of distal repressors sites showed strong nucleosome positioning on both sides (Additional file 7). All these results were consistent with our previous analyses. However, the asymmetric nucleosome occupancy patterns were neglected 

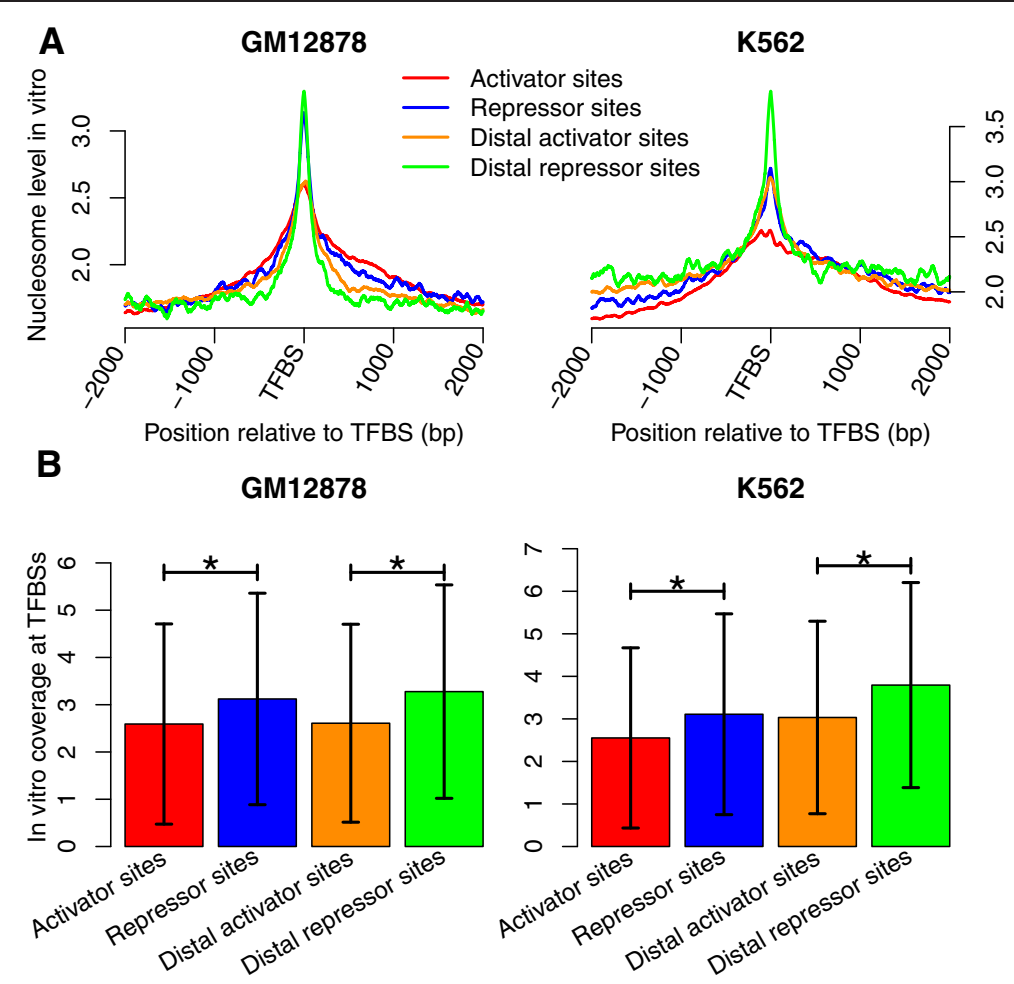

Figure 4 Repressor binding sequences are more likely to encode nucleosomes intrinsically. (A) In vitro nucleosome occupancy around the activator and repressor sites, and their distal sites in both GM12878 and K562 cells. (B) Average in vitro nucleosome occupancy at the activator and repressor sites, and their distal sites. The in vitro nucleosome signals were normalized by the length of TFBSs and given as the mean \pm standard deviation. Statistically significant differences were detected using a Wilcoxon rank-sum test. $\left(^{*}\right) P<2.2 \times 10^{-16}$.

in our previous analyses. The cluster analysis of nucleosome signals around TFBSs indicated that the majority of TFBSs showed strong nucleosome positioning on one side, suggesting that asymmetric patterns of nucleosome occupancy were more pervasive around TFBSs.

\section{Nucleosome occupancy patterns correlate with gene expression}

It has been suggested that nucleosome occupancy patterns around TSSs are associated with gene expression $[29,30]$. Here we assigned each of the binding sites to the nearest gene based on its distance to TSSs [31,32], and investigated the correlations between in vivo nucleosome occupancy patterns around TFBSs and gene expression. First, we grouped the nucleosome occupancy signals of all TFBSs into three clusters using the CAGT software, and examined the expression levels of unique target genes for each of the clusters in both GM12878 and K562 cells (Figure 8). Increasing the number of clusters would lead to a decrease in the number of target genes for each cluster, and we empirically grouped into three clusters. TFBSs in the first cluster in both GM12878 and K562 cells showed fuzzy nucleosome positioning and relatively higher nucleosome occupancy on one side $\left(P_{-} 1\right)$; TFBSs in the second cluster had strongly positioned nucleosomes on both sides (P_2); and TFBSs in the third cluster were occupied by nucleosomes (P_3). Analysis of gene expression levels for each of the clusters in both GM12878 and K562 cells indicated that TFBSs in the first cluster were associated with genes that were in general significantly more highly expressed (Figure $8 \mathrm{~B}$ and D; Wilcoxon rank-sum test, $P<3.7 \times 10^{-16}$ ).

Activators and repressors have the opposite effects on gene expression, but the binding of both of them can generate similar patterns of nucleosome occupancy (Additional file 7). In order to clearly demonstrate the correlations between TF binding and gene expression, we further clustered the nucleosome occupancy signals around activator and repressor binding sites using the CAGT software, and examined their relevance to gene expression in both GM12878 and K562 cells. Nucleosome signals around proximal activator binding sites in both GM12878 and K562 cells were first grouped into three clusters using the CAGT software (Figure 9A and B). Pattern 1 ( $\mathrm{P} \_1$ ) in both GM12878 and K562 cells showed fuzzy nucleosome positioning and higher nucleosome occupancy on one side of TFBSs; Pattern 2 (P_2) in GM12878 and Pattern 3 (P_3) in K562 showed high nucleosome occupancy at TFBSs; Pattern 3 (P_3) in GM12878 and Pattern 2 (P_2) in K562 exhibited one 

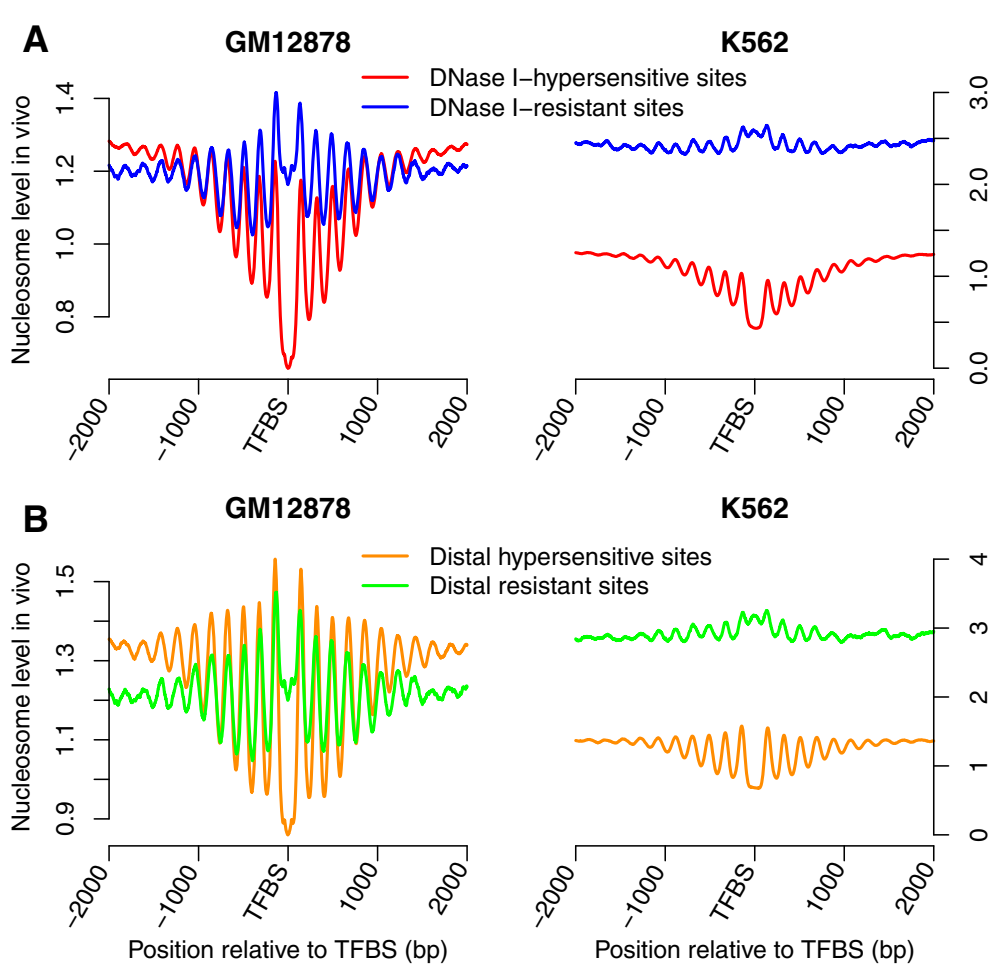

K562 I-hypersensitive and -resistant binding sites in both GM12878 and K562 cells. (B) In vivo nucleosome occupancy around distal DNase I-hypersensitive and -resistant binding sites.

well-positioned nucleosome on one side of TFBSs. The analysis of target gene expression for each cluster of proximal activator sites indicated that genes associated with proximal activator sites in Pattern 2 had significantly lower expression in the GM12878 cell line (Figure 10A; Wilcoxon rank-sum test, $P<0.002$ ), and genes associated with sites in Pattern 3 had significantly lower expression in the K562 cell line (Figure 10B; $P<0.007$ ). The same analysis for the proximal repressor binding sites indicated that genes associated with proximal repressor sites in Pattern 3 (P_3) had significantly lower expression in the GM12878 cell line (Figure 10C; Wilcoxon rank-sum test, $P<0.0006)$. Pattern 3 in GM12878 showed wellpositioned nucleosomes on both sides of proximal repressor sites (Figure 9C). We observed no significant differences in the expression levels of target genes for different clusters of proximal repressor sites in the K562 cell line (Figure 9D and Figure 10D). We also performed the same analyses on the distal activator and repressor binding sites in both GM12878 and K562 cells, and found no significant differences in the expression levels of target genes for different clusters of distal activator and repressor sites (Additional file 8 and Additional file 9). This might result from the assignment of target genes. We simply assigned target genes using the nearest distance, and it would be unreliable for the binding sites distal to TSSs.

\section{Discussion}

Transcription is regulated by the dynamic binding of TFs to the underlying DNA sequences in a cell type-specific manner [3]. Most eukaryotic genomic DNA is packaged into nucleosomes, and TF binding is thus strongly associated with the local chromatin structure surrounding TFBSs $[4,5]$. Chromatin can affect the recognition and binding of TFs; TFs can, in turn, direct the chromatin remodeling complexes to their target regions [2]. We examined the nucleosome occupancy profiles around TFBSs to better understand the intricate relationships between TF binding and chromatin structure, and we also investigated the correlations between binding sites with different patterns of nucleosome occupancy and gene expression.

Although previous studies have demonstrated the nucleosome occupancy profiles around the binding sites for several specific TFs, our findings expand the current knowledge of nucleosome occupancy at TFBSs, based on the greater number of TFs. First, TF binding regions are generally nucleosome-depleted as a result of TF and nucleosome interactions. TFs can directly compete with 


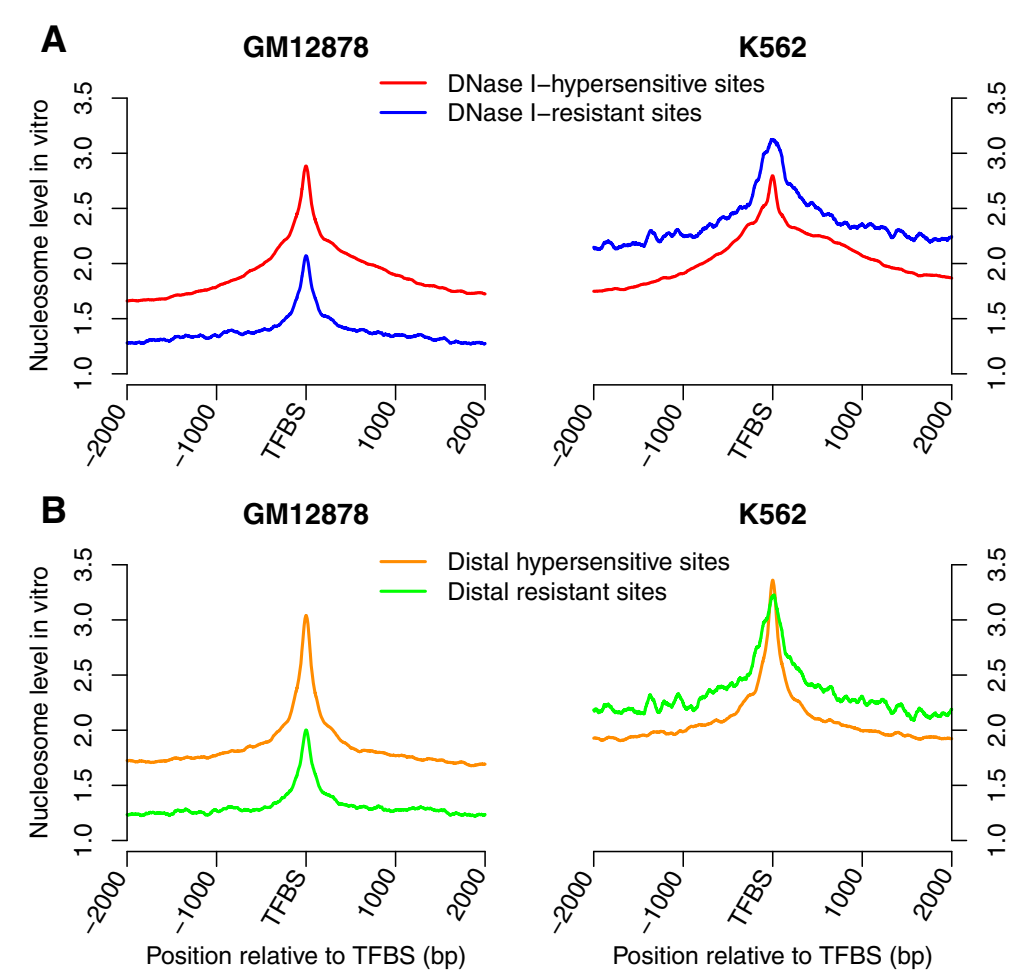

Figure 6 DNase I-resistant binding regions are more likely to form nucleosomes in the K562 cell line. (A) In vitro nucleosome occupancy around DNase I-hypersensitive and -resistant sites in both GM12878 and K562 cells. (B) In vitro nucleosome occupancy around distal DNase I-hypersensitive and -resistant sites.

nucleosomes and evict them from the DNA, while some TFs are aided by pioneer factors to bind to DNA [6-8]. Pioneer factors, such as FoxA, GATA, and PU.1, can bind to nucleosomal DNA and displace nucleosomes to help other TFs access their sites [33]. Although nucleosome-depleted regions are necessary for most TFBSs, some TFs may bind to nucleosomal DNA without nucleosome reorganization. Previous studies have suggested that TF NF-kB p50 can bind to nucleosomal DNA without perturbing the overall structure of the nucleosome [34]. We found that many TFBSs were located within DNase-I resistant regions, and these DNase-I resistant sites were cell-type specific. The cluster analysis using the CAGT software also indicated that a small proportion of TFBSs were indeed occupied by nucleosomes. It should be also noted that TFs might directly bind to sites in the nucleosome-depleted regions, especially in the proximal promoter near TSSs. Second, betterpositioned nucleosomes were observed around the repressor sites compared with those around the activator sites. Repressors were more likely to bind to nucleosomal DNA, which might require catalyzed remodeling, in the human genome. The higher dependence on chromatin remodeling complexes might contribute to the stronger nucleosome positioning around the repressor sites. Besides, repressors are more associated with closed chromatin compared with activators. The highly positioned nucleosomes might result from the recruitment of different chromatin remodelers. Third, although a quarter of TFBSs showed arrays of well-positioned nucleosomes on both sides, the majority of TFBSs exhibited one or more wellpositioned nucleosomes on one side, and a small proportion of TFBSs were occupied by nucleosomes in vivo. Correlating these different patterns of nucleosome occupancy with the expression levels of target genes indicated that genes with TFBSs exhibiting well-positioned nucleosomes on both sides or occupied by nucleosomes, had significantly lower expression levels. The analysis of gene expression for proximal activator and repressor binding sites further indicated that genes with repressor sites exhibiting well-positioned nucleosomes on both sides, and genes with activator sites occupied by nucleosomes had significantly lower expression, suggesting that actions of activators and repressors are associated with the nucleosome occupancy around their binding sites.

\section{Conclusions}

The DNA sequence, TF binding and chromatin remodeling events are important determinants of in vivo nucleosome organization in human cells. In this study, we 


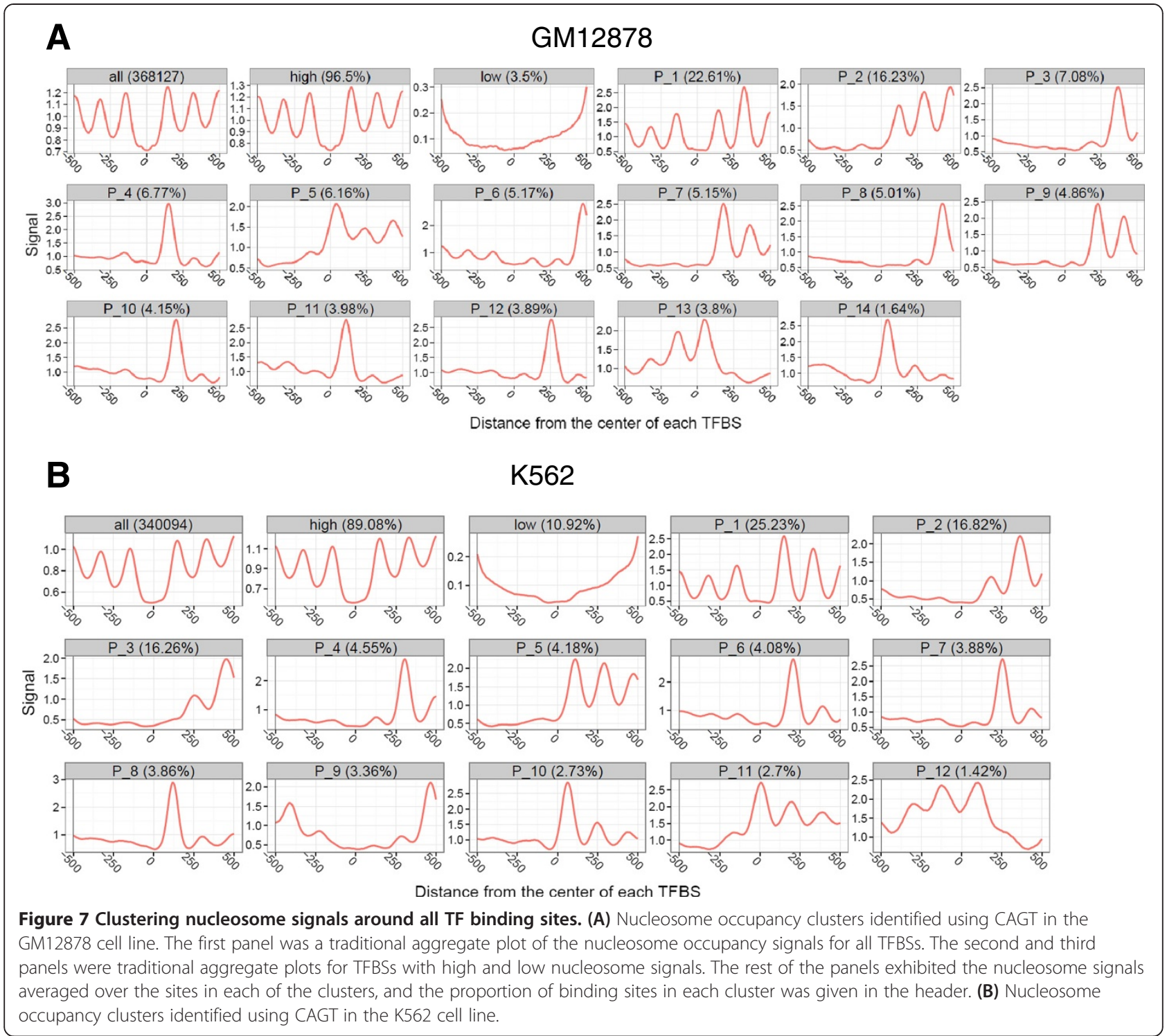

systematically investigated the nucleosome occupancy profiles around TFBSs and their relevance to gene expression in human GM12878 and K562 cells. The nucleosomes were generally depleted at TFBSs in vivo, and asymmetric patterns of nucleosome occupancy were more pervasive around TFBSs. However, approximately a quarter of TFBSs showed well-positioned nucleosomes on both sides, and a small proportion of TFBSs were occupied by nucleosomes. Compared with the distal sites, proximal sites showed fuzzier nucleosome positioning. These proximal sites were located within $1 \mathrm{~kb}$ of TSSs, and RNA polymerase complexes near the TSSs had a large effect on the nucleosome occupancy distributions around these sites. Compared with activator sites, nucleosomes around repressor sites were better positioned. In addition, nucleosome occupancy patterns around TFBSs were correlated with the expression levels of target genes. Genes with repressor sites exhibiting well-positioned nucleosomes on both sides, and genes with activator sites occupied by nucleosomes had significantly lower expression.

\section{Methods}

\section{Data source}

The aligned MNase-seq reads for in vivo nucleosomes in both GM12878 and K562 cells [29], were generated by the Snyder lab and downloaded from University of California Santa Cruz (UCSC) Genome Browser FTP server (ftp://hgdownload.soe.ucsc.edu/goldenPath/hg19/ encodeDCC/). Downloaded files contained reads mapped to the hg19 human reference genome in the BAM format, and we therefore converted these to BED format using 


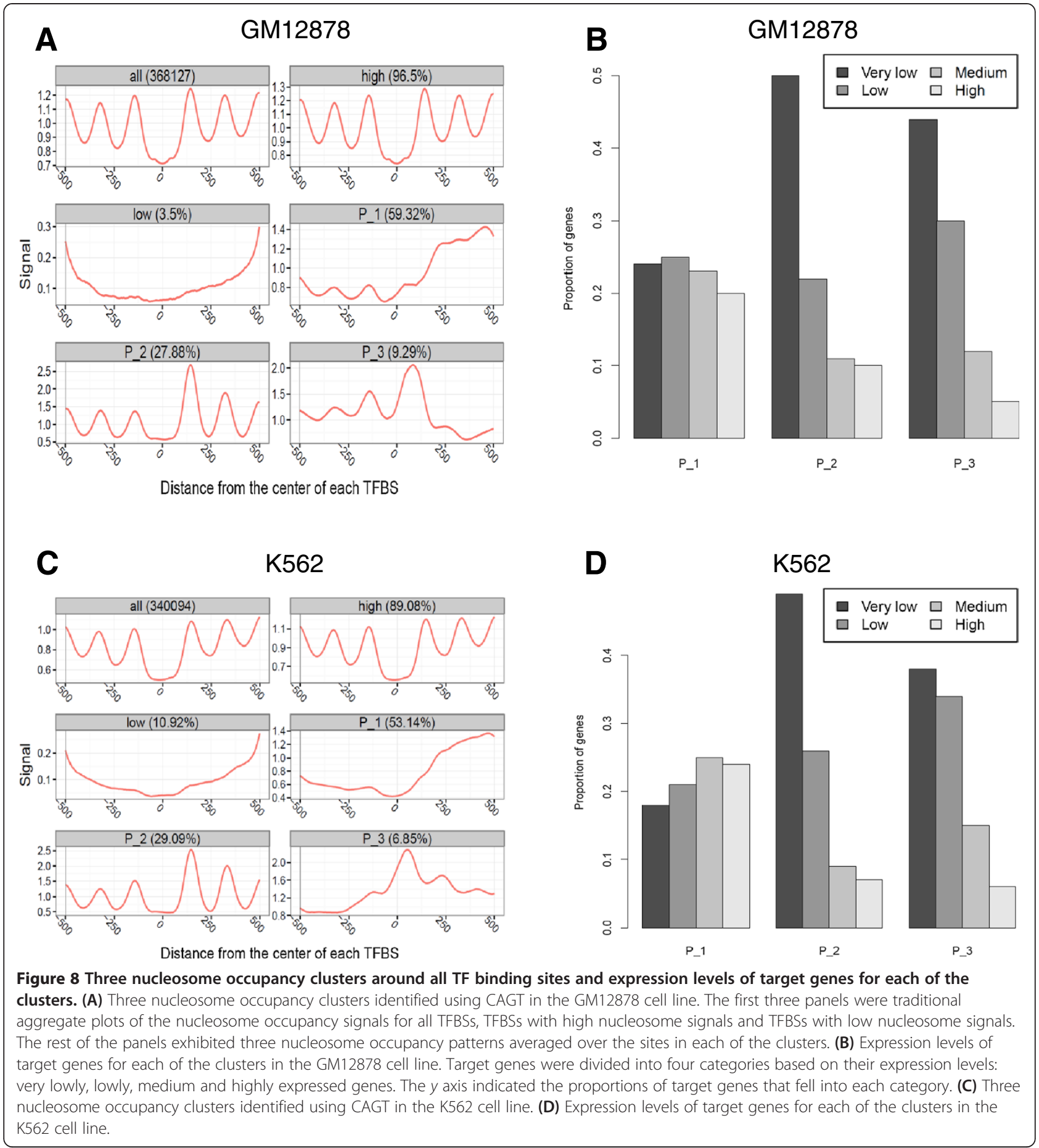

BEDTools [35]. We further removed duplicate reads that were exactly mapped to the same position, as these reads might arise from biases during ChIP-DNA amplification and sequencing library preparation [36]. In order to determine the in vivo nucleosome occupancy level at each genomic coordinate, we shifted the start position of each read by $73 \mathrm{bp}$ in the $5^{\prime}$ to $3^{\prime}$ direction, and counted the total number of reads with a window size of $60 \mathrm{bp}$ on both strands. These counts were finally normalized by the expected number of reads in the 60-bp window, which was calculated as:

(Number of mapped reads) $\times 60 /($ genome size $)$.

The in vitro nucleosomes were assembled through combining the human genomic DNA with recombinantly 


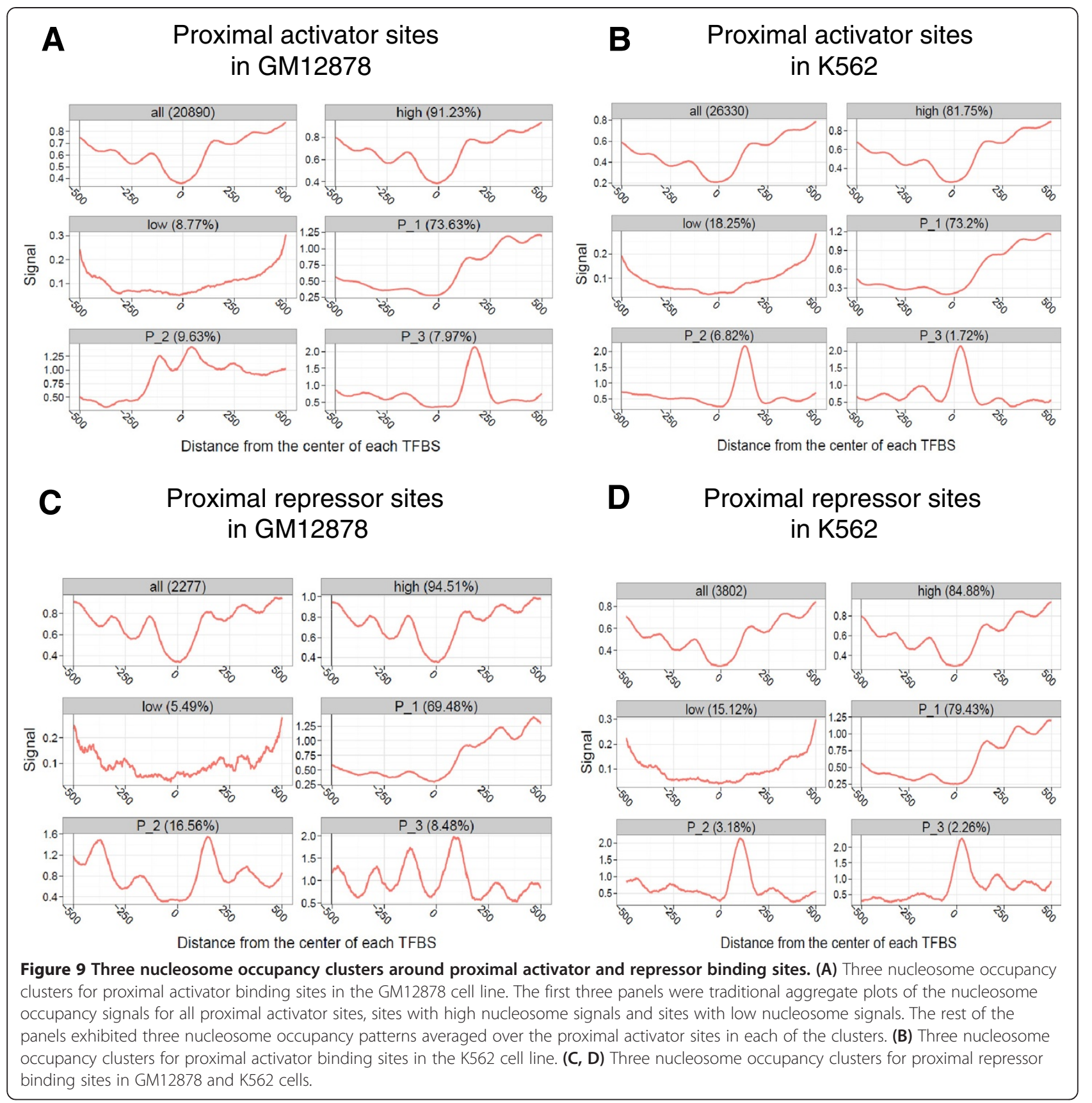

derived histone octamers [13], and the raw sequenced reads for in vitro nucleosomes were obtained from the NCBI Gene Expression Omnibus (http://www.ncbi.nlm. nih.gov/geo/) using the accession number GSE25133. The sequenced reads were first mapped to the hg19 human genome using the Bowtie aligner [37], allowing a maximum of two mismatches. Then duplicate mapped reads were removed and the rest of reads were shifted by $73 \mathrm{bp}$ in the $5^{\prime}$ to $3^{\prime}$ direction. Reads within a 60-bp window were finally counted and normalized to construct the in vitro nucleosome occupancy profile along the human genome, as in the processing of in vivo nucleosome reads.

The peaks of enriched signals in DNase I hypersensitivity experiments [38], generated by the Crawford lab, were downloaded from the UCSC FTP server. Crawford's group mapped DNase-seq reads to the hg19 human genome using the BWA aligner [39], calculated the signal enrichment at each genomic coordinate using the FSeq software [40], and identified peaks from the F-Seq density signals. These DNase I-hypersensitive regions 

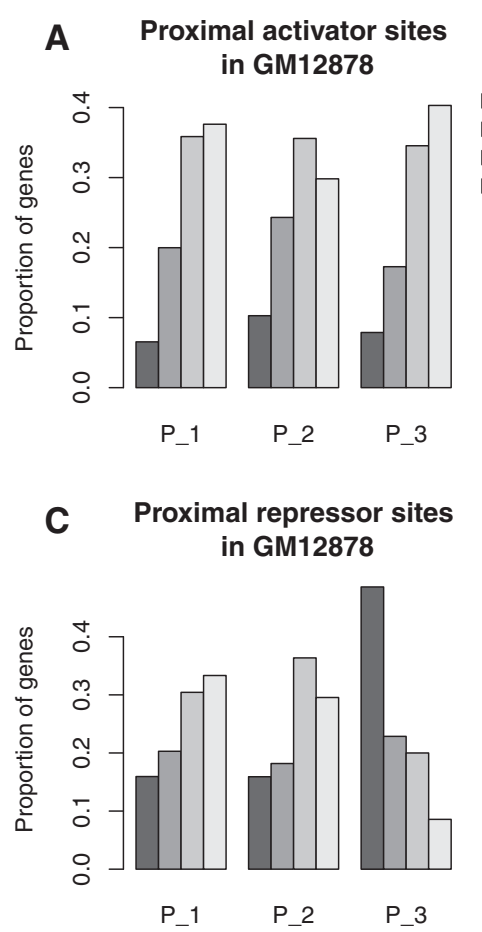
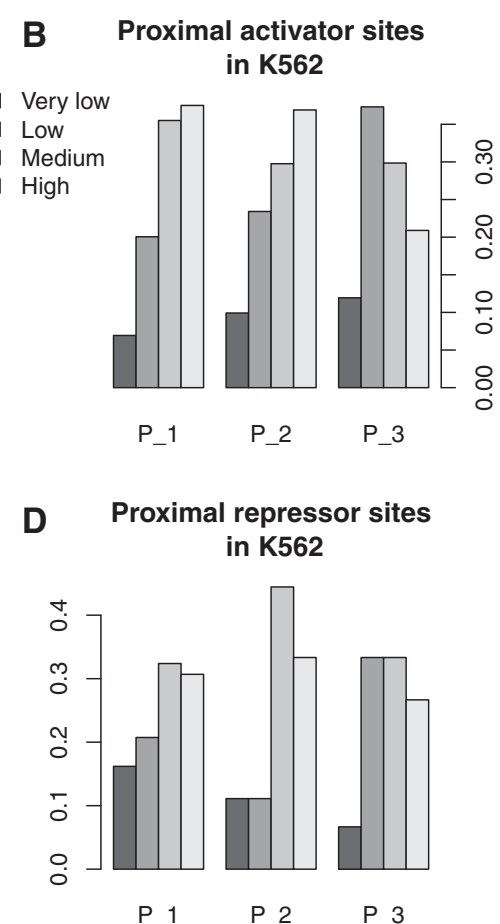

Figure 10 Expression levels of target genes for each cluster of proximal activator and repressor binding sites. (A) Expression levels of target genes for each cluster of proximal activator binding sites in the GM12878 cell line. Genes associated with binding sites in Pattern 2 had significantly lower expression. (B) Expression levels of target genes for each cluster of proximal activator binding sites in the K562 cell line. Genes associated with sites in Pattern 3 had significantly lower expression. (C) Expression levels of target genes for each cluster of proximal repressor binding sites in the GM1 2878 cell line. Genes associated with proximal repressor sites in Pattern 3 had significantly lower expression. (D) Expression levels of target genes for each cluster of proximal repressor binding sites in the K562 cell line. Statistically significant differences were detected using a Wilcoxon rank-sum test.

reflected the openness of the chromatin and the accessibility of the genome in GM12878 and K562 cells.

TF binding sites for 519 binding motifs estimated with CENTIPEDE [19] were downloaded from http://centipede.uchicago.edu/SimpleMulti/. The total numbers of binding sites in the GM12878 and K562 cells were 368,127 and 340,094, respectively. The initial downloaded data were mapped to the hg18 human reference genome, and the binding locations were therefore converted from hg18 to hg19 using liftOver, provided by the UCSC Genome Browser. CENTIPEDE scanned the human genome with a TF binding motif to obtain candidate binding sites and computed a posterior probability for each candidate site to identify the real binding sites. It should be noted that although CENTIPEDE predicted binding sites using 519 TF binding motifs, only 220 and 260 binding motifs (Additional file 10) were included in the GM12878 and K562 cells, respectively, to ensure that each of the binding sites in downloaded files had a posterior probability greater than 0.999 .

The aligned RNA-seq reads in GM12878 and K562 cells [41], were generated by the Caltech and downloaded from the UCSC FTP server. These paired-end reads were aligned to the hg19 genome and stored in the BAM format. The hg19 RefSeq gene annotation data [42] were also obtained from the UCSC FTP server. In order to determine the TSS position and the expression level of each of the genes, we first removed non-protein-coding transcripts from the hg19 RefSeq file. Then, we used the transcript assembly and quantification software Cufflinks [43] with default settings to calculate the expression value of each RefSeq transcript, which was quantified in fragments per kilobase of exon per million mapped fragments [44]. For alternatively spliced transcripts encoding the same protein, only the transcript with the highest expression value was used. A total of 19,019 TSSs of RefSeq genes in both GM12878 and K562 cells were obtained to investigate the nucleosome occupancy around TSSs and define the distance between a binding site and the nearest TSS. We further classified genes into four categories on the basis of their expression levels. Genes with expression levels less than the first quartile, between the first and second quartiles, between the second and third quartiles, and greater than the third quartile were considered as very lowly, lowly, medium and highly expressed genes, respectively. 


\section{Nucleosome occupancy around TF binding sites}

For a group of CENTIPEDE sites, we extracted the nucleosome signal in a \pm 2 - $\mathrm{kb}$ window around each binding site and averaged nucleosome signals over all sites to represent the nucleosome occupancy around the binding sites. In addition, considering the confounding factors of nearby TSSs, we assigned each of the binding sites to the nearest gene based on its distance to the TSSs and reversed the shape profile of binding sites on the negative strand before averaging, to avoid a misleading aggregation.

\section{Identification of activators and repressors}

All activators and repressors were first retrieved from the UniProt database [26], a comprehensive resource for protein sequence and annotation data, to determine whether a TF was an activator or repressor. The search terms for activators and repressors were "activator AND organism:human AND reviewed:yes" and "repressor AND organism:human AND reviewed:yes", respectively. Some multifunctional TFs, such as YY1 and CTCF, were annotated as both activators and repressors and were further removed in the analysis. We finally identified 20 activators and four repressors in the GM12878 cell line, and 25 activators and six repressors in the K562 cell line (Additional file 2).

\section{Clustering nucleosome occupancy around TF binding sites}

We extracted the nucleosome signals in a \pm 500 -bp window around each binding site, and clustered these nucleosome signals using the CAGT software [29]. CAGT uses the $k$-medians algorithm to obtain a relatively large number of compact clusters, and then redundant clusters are merged using the hierarchical agglomerative clustering. The number of clusters was set to 40 and a correlationbased distance function was used in the $k$-medians clustering in our analyses. Hierarchical agglomerative clustering iteratively merged the two most similar clusters and mirror clusters were also merged. If the number of clusters was set to 1 , a distance threshold was set to 0.4 and two closest clusters with a distance below the threshold would be merged in the hierarchical agglomerative clustering. In addition, binding sites, whose nucleosome signal profiles had variance below a threshold of 0.1 , were removed prior to the $k$-medians clustering.

\section{Additional files}

Additional file 1: 519 TF binding motifs and their corresponding TFs. The list included 519 TF binding motifs and their corresponding TFs. One TF may have more than one binding motifs.

Additional file 2: Activators and repressors in GM12878 and K562 cells. The list included 20 activators and four repressors in GM12878 cells, and 25 activators and six repressors in $\mathrm{K} 562$ cells, which were identified by querying the UniProt database.

Additional file 3: Coordinates of the distal activator and repressor binding sites in GM12878 and K562 cells. The four sheets contained the coordinates of 24,133 distal activator sites and 3,422 distal repressor sites in the GM12878 cell line, and 13,840 distal activator sites and 2,489 distal repressor sites in the $\mathrm{K} 562$ cell line.

Additional file 4: In vivo nucleosome occupancy around the distal binding sites for each of activators.

Additional file 5: In vivo nucleosome occupancy around the distal binding sites for each of repressors.

Additional file 6: Clustering nucleosome signals around proximal and distal binding sites. (A, B) Nucleosome occupancy clusters around proximal sites in GM12878 and K562 cells. (C, D) Nucleosome occupancy clusters around distal sites in GM12878 and K562 cells.

Additional file 7: Clustering nucleosome signals around distal activator and repressor binding sites. (A, B) Nucleosome occupancy clusters around distal activator sites in GM12878 and K562 cells. (C, D) Nucleosome occupancy clusters around distal repressor sites in GM12878 and K562 cells.

Additional file 8: Three nucleosome occupancy clusters around distal activator and repressor binding sites. (A, B) Three nucleosome occupancy clusters for distal activator binding sites in GM12878 and K562 cells. (C, D) Three nucleosome occupancy clusters for distal repressor binding sites in GM12878 and K562 cells.

Additional file 9: Expression levels of target genes for each cluster of distal activator and repressor binding sites. (A, B) Expression levels of target genes for each cluster of distal activator binding sites in GM12878 and K562 cells. (C, D) Expression levels of target genes for each cluster of distal repressor binding sites in GM12878 and K562 cells.

Additional file 10: TF binding motifs in GM12878 and K562 cells. The list included 220 motifs in the GM12878 cell line and 260 motifs in the K562 cell line. Each of the binding sites for these motifs had a posterior probability greater than 0.999 .

\section{Abbreviations}

TF: Transcription factor; TFBS: Transcription factor binding site; TSS: Transcription start site; CAGT: Clustered AGgregation tool.

\section{Competing interests}

The authors declare that they have no competing interests.

\section{Authors' contributions}

YN and XS conceived and designed the study. YN, XC and JC acquired and analyzed the data. YN interpreted the results and drafted the manuscript. XS consulted on the approaches and revised the manuscript. All authors have read and approved the final manuscript.

\section{Acknowledgements}

We thank Hongde Liu for discussions on the statistical analysis. We thank the two anonymous reviewers for their valuable suggestions. This work was supported by the National Basic Research Program of China (2012CB316501) and the National Natural Science Foundation of China (61073141)

Received: 24 December 2013 Accepted: 10 June 2014

Published: 19 June 2014

\section{References}

1. Lenhard B, Sandelin A, Carninci P: Metazoan promoters: emerging characteristics and insights into transcriptional regulation. Nat Rev Genet 2012, 13(4):233-245.

2. van Bakel $\mathrm{H}$ : Interactions of transcription factors with chromatin. Subcell Biochem 2011, 52:223-259.

3. Pan $Y$, Tsai CJ, Ma B, Nussinov R: Mechanisms of transcription factor selectivity. Trends Genet 2010, 26(2):75-83.

4. lyer VR: Nucleosome positioning: bringing order to the eukaryotic genome. Trends Cell Biol 2012, 22(5):250-256. 
5. Bai L, Morozov AV: Gene regulation by nucleosome positioning. Trends Genet 2010, 26(11):476-483.

6. Wang X, Bai L, Bryant GO, Ptashne M: Nucleosomes and the accessibility problem. Trends Genet 2011, 27(12):487-492

7. He HH, Meyer CA, Shin H, Bailey ST, Wei G, Wang Q, Zhang Y, Xu K, Ni M, Lupien M, Mieczkowski P, Lieb JD, Zhao K, Brown M, Liu XS: Nucleosome dynamics define transcriptional enhancers. Nat Genet 2010, 42(4):343-347.

8. Segal E, Widom J: What controls nucleosome positions? Trends Genet 2009, 25(8):335-343.

9. Schones DE, Cui K, Cuddapah S, Roh TY, Barski A, Wang Z, Wei G, Zhao K: Dynamic regulation of nucleosome positioning in the human genome. Cell 2008, 132(5):887-898.

10. Hughes AL, Jin Y, Rando OJ, Struhl K: A functional evolutionary approach to identify determinants of nucleosome positioning: a unifying model for establishing the genome-wide pattern. Mol Cell 2012, 48(1):5-15.

11. Fu $Y$, Sinha $M$, Peterson $C L$, Weng $Z$ : The insulator binding protein $C T C F$ positions 20 nucleosomes around its binding sites across the human genome. PLOS Genet 2008, 4(7):e1000138.

12. Cuddapah S, Jothi R, Schones DE, Roh TY, Cui K, Zhao K: Global analysis of the insulator binding protein CTCF in chromatin barrier regions reveals demarcation of active and repressive domains. Genome Res 2009, 19(1):24-32

13. Valouev A, Johnson SM, Boyd SD, Smith CL, Fire AZ, Sidow A: Determinants of nucleosome organization in primary human cells. Nature 2011, 474(7352):516-520.

14. Wang J, Zhuang J, lyer S, Lin X, Whitfield TW, Greven MC, Pierce BG, Dong X, Kundaje A, Cheng Y, Rando OJ, Birney E, Myers RM, Noble WS, Snyder M, Weng Z: Sequence features and chromatin structure around the genomic regions bound by 119 human transcription factors. Genome Res 2012, 22(9):1798-1812.

15. Wilson D, Charoensawan V, Kummerfeld SK, Teichmann SA: DBDtaxonomically broad transcription factor predictions: new content and functionality. Nucleic Acids Res 2008, 36(Database issue):D88-92.

16. Vaquerizas JM, Kummerfeld SK, Teichmann SA, Luscombe NM: A census of human transcription factors: function, expression and evolution. Nat Rev Genet 2009, 10(4):252-263.

17. Tillo D, Kaplan N, Moore IK, Fondufe-Mittendorf Y, Gossett AJ, Field Y, Lieb JD, Widom J, Segal E, Hughes TR: High nucleosome occupancy is encoded at human regulatory sequences. PLoS One 2010, 5(2):e9129.

18. Ernst J, Plasterer HL, Simon I, Bar-Joseph Z: Integrating multiple evidence sources to predict transcription factor binding in the human genome. Genome Res 2010, 20(4):526-536.

19. Pique-Regi R, Degner JF, Pai AA, Gaffney DJ, Gilad Y, Pritchard JK: Accurate inference of transcription factor binding from DNA sequence and chromatin accessibility data. Genome Res 2011, 21(3):447-455.

20. Struhl K, Segal E: Determinants of nucleosome positioning. Nat Struct Mo Biol 2013, 20(3):267-273.

21. Huebert DJ, Kuan PF, Keles S, Gasch AP: Dynamic changes in nucleosome occupancy are not predictive of gene expression dynamics but are linked to transcription and chromatin regulators. Mol Cell Biol 2012, 32(9):1645-1653.

22. Maston GA, Evans SK, Green MR: Transcriptional regulatory elements in the human genome. Annu Rev Genomics Hum Genet 2006, 7:29-59.

23. Brogaard $K, X i L$, Wang JP, Widom J: A map of nucleosome positions in yeast at base-pair resolution. Nature 2012, 486(7404):496-501.

24. Gossett AJ, Lieb JD: In vivo effects of histone $\mathrm{H} 3$ depletion on nucleosome occupancy and position in Saccharomyces cerevisiae. PLoS Genet 2012, 8(6):e1002771

25. Charoensawan V, Janga SC, Bulyk ML, Babu MM, Teichmann SA: DNA sequence preferences of transcriptional activators correlate more strongly than repressors with nucleosomes. Mol Cell 2012, 47(2):183-192.

26. The UniProt Consortium: Reorganizing the protein space at the universal protein resource (UniProt). Nucleic Acids Res 2012, 40(Database issue):D71-75.

27. Burns LG, Peterson CL: The yeast SWI-SNF complex facilitates binding of a transcriptional activator to nucleosomal sites in vivo. Mol Cell Biol 1997, 17(8):4811-4819.

28. Bai L, Charvin G, Siggia ED, Cross FR: Nucleosome-depleted regions in cell-cycle-regulated promoters ensure reliable gene expression in every cell cycle. Dev Cell 2010, 18(4):544-555.

29. Kundaje A, Kyriazopoulou-Panagiotopoulou S, Libbrecht M, Smith CL, Raha D, Winters EE, Johnson SM, Snyder M, Batzoglou S, Sidow A: Ubiquitous heterogeneity and asymmetry of the chromatin environment at regulatory elements. Genome Res 2012, 22(9):1735-1747.

30. Lee W, Tillo D, Bray N, Morse RH, Davis RW, Hughes TR, Nislow C: A high-resolution atlas of nucleosome occupancy in yeast. Nat Genet 2007, 39(10):1235-1244

31. Zhu LJ, Gazin C, Lawson ND, Pages H, Lin SM, Lapointe DS, Green MR: ChIPpeakAnno: a bioconductor package to annotate ChIP-seq and ChIP-chip data. BMC Bioinformatics 2010, 11:237.

32. Yanez-Cuna JO, Dinh HQ, Kvon EZ, Shlyueva D, Stark A: Uncovering cis-regulatory sequence requirements for context-specific transcription factor binding. Genome Res 2012, 22(10):2018-2030.

33. Zaret KS, Carroll JS: Pioneer transcription factors: establishing competence for gene expression. Genes Dev 2011, 25(21):2227-2241.

34. Angelov D, Lenouvel F, Hans F, Muller CW, Bouvet P, Bednar J, Moudrianakis EN, Cadet J, Dimitrov S: The histone octamer is invisible when NF-kappaB binds to the nucleosome. J Biol Chem 2004, 279(41):42374-42382.

35. Quinlan AR, Hall IM: BEDTools: a flexible suite of utilities for comparing genomic features. Bioinformatics 2010, 26(6):841-842.

36. Zhang Y, Liu T, Meyer CA, Eeckhoute J, Johnson DS, Bernstein BE, Nusbaum C, Myers RM, Brown M, Li W, Liu XS: Model-based analysis of ChIP-Seq (MACS). Genome Biol 2008, 9(9):R137.

37. Langmead B, Trapnell C, Pop M, Salzberg SL: Ultrafast and memoryefficient alignment of short DNA sequences to the human genome. Genome Biol 2009, 10(3):R25.

38. Thurman RE, Rynes E, Humbert R, Vierstra J, Maurano MT, Haugen E, Sheffield NC, Stergachis AB, Wang H, Vernot B, Garg K, John S, Sandstrom R, Bates D, Boatman L, Canfield TK, Diegel M, Dunn D, Ebersol AK, Frum T, Giste E, Johnson AK, Johnson EM, Kutyavin T, Lajoie B, Lee BK, Lee K, London D, Lotakis D, Neph S, et al: The accessible chromatin landscape of the human genome. Nature 2012, 489(7414):75-82.

39. Li H, Ruan J, Durbin R: Mapping short DNA sequencing reads and calling variants using mapping quality scores. Genome Res 2008 , 18(11):1851-1858.

40. Boyle AP, Guinney J, Crawford GE, Furey TS: F-Seq: a feature density estimator for high-throughput sequence tags. Bioinformatics 2008, 24(21):2537-2538.

41. Djebali S, Davis CA, Merkel A, Dobin A, Lassmann T, Mortazavi A, Tanzer A Lagarde J, Lin W, Schlesinger F, Xue C, Marinov GK, Khatun J, Williams BA, Zaleski C, Rozowsky J, Roder M, Kokocinski F, Abdelhamid RF, Alioto T, Antoshechkin I, Baer MT, Bar NS, Batut P, Bell K, Bell I, Chakrabortty S, Chen $X$, Chrast J, Curado J, et al: Landscape of transcription in human cells. Nature 2012, 489(7414):101-108.

42. Pruitt KD, Tatusova T, Maglott DR: NCBI reference sequence (RefSeq): a curated non-redundant sequence database of genomes, transcripts and proteins. Nucleic Acids Res 2005, 33(Database issue):D501-504.

43. Trapnell C, Williams BA, Pertea G, Mortazavi A, Kwan G, van Baren MJ, Salzberg SL, Wold BJ, Pachter L: Transcript assembly and quantification by RNA-Seq reveals unannotated transcripts and isoform switching during cell differentiation. Nat Biotechnol 2010, 28(5):511-515.

44. Mortazavi A, Williams BA, McCue K, Schaeffer L, Wold B: Mapping and quantifying mammalian transcriptomes by RNA-Seq. Nat Methods 2008, 5(7):621-628.

doi:10.1186/1471-2164-15-493

Cite this article as: Nie et al:: Nucleosome organization in the vicinity of transcription factor binding sites in the human genome. BMC Genomics 2014 15:493 